\title{
Wet-etched phononic crystal waveguiding on GaAs
}

Edward Muzar, Golnaz Azodi Aval, James A. H. Stotz

Department of Physics, Engineering Physics, \& Astronomy,

Queen's University, Kingston, Ontario, Canada

\section{Abstract}

A wet-etched phononic crystal waveguide in GaAs with approximately two micron deep inclusions is studied both numerically and experimentally for controlled surface acoustic wave propagation. Numerically, the phononic crystal was modelled using the finite element method (FEM) with COMSOL Multiphysics, and the surface displacement of the acoustic waves was measured using optical interferometry. The computed filter response of the phononic crystal confirmed that the phononic crystal was an effective stop band filter in the interval of $400 \mathrm{MHz}$ and $450 \mathrm{MHz}$. An L1 linear defect waveguide with a stepped funnel entrance design is shown to perform well at a surface acoustic wave frequency of 410.344 MHz and in agreement to simulated results. The phononic crystal waveguide system shows promise for use in acoustic control of GaAs-based quantum nanostructures.

\section{Introduction}

Quantum semiconductor systems based on GaAs have been of interest for several decades (Stormer, et al., 1979), and the interest in nanostructures coupled with excellent material quality have made GaAs a standard, prototypical system in which to work with quantum interactions in the solid state. Expanding on the developments in GaAs-based nanostructures, surface acoustic waves (SAWs) were applied to quantum systems to achieve enhanced functionality in quantum wells (Wixforth, et al., 1986) (Shilton, et al., 1996), quantum wires, and quantum dots (Couto Jr., et al., 2009). These systems used plane-wave SAWs to achieve their objectives, but there is potential to better control SAWs and target them to particular regions of a device using acoustic waveguides. Considering the many interesting structures and application using photonic crystal waveguides, phononic crystal waveguides may be one alternative to control acoustic modulations on a chip. In GaAs, phononic crystals with finite-depth wet-etch inclusions have been shown to filter and reflect SAWs effectively for bandgap frequencies (Petrus, et al., 2014). The interest is then on adding phononic crystal lattice defects to facilitate waveguiding behaviour (Olsson III \& El-Kady, 2009). Single linear defect with bending waveguides have been shown to propagate a travelling wave (Khelif, et al., 2004) and have a well-defined dispersion (Pennec, et al., 2010). In this paper, three-dimensional finite 
element method (FEM) modelling is used to simulate transmission characteristics of a GaAs-based phononic crystal composed of a two-dimensional array of wet-etched inclusions and a single linear defect waveguide with a funnel entrance. Three-dimensional FEM has previously been utilized to demonstrate bandgap formation in phononic crystals on a piezoelectric material (Yudistira, et al., 2012). Our simulations are then compared with an operational waveguide device at a SAW frequency within the bandgap and on the modal resonance band. The device is characterized by mapping the acoustic vertical displacement amplitude on the surface of the phononic crystal device using optical interferometry.

\section{Computational and Experimental Procedures}

Modelling of the phononic crystal structures was performed using three-dimensional FEM simulations were completed in COMSOL Multiphysics. A 10 micron deep (001) GaAs substrate domain was used, and the phononic crystal was defined by a square lattice with a single inclusion basis. The phononic crystal filter response for the $\Gamma-X$ direction wavevectors, which are parallel to the [110] GaAs crystal directions, was determined using a direct solver in the frequency domain. The physics model consisted of linear elasticity and electrostatics with coupling via the piezoelectric effect. SAWs were generated by applying a one volt amplitude harmonic potential difference between a grounded electrode and an applied harmonic potential electrode. The electrodes, parallel to [110] on the surface $(z=10 \mu \mathrm{m})$, were modelled as surface boundary conditions in the electrostatics physics computation. The phononic crystal inclusion geometry is seen in Figure 1(a). It is a composite geometry formed from three truncated cones. One comprises the base structure of a slanted wall cylinder. Two others (inverted from one another) are stretched by a factor of 1.1 in the $\mathrm{x}$-direction give the subsurface corner feature. The etch geometry was estimated based on a GaAs wet-etch study with hydrogen peroxide and citric acid (DeSalvo, et al., 1992). The inclusion size was specified by setting the circular face of the cone at surface-level at a filling fraction of 0.6 before subsequent stretching yielding a final surface filling fraction of 0.66 . The volume fraction of this same geometry was 0.52 considering up to the total depth of the inclusion of $2 \mu \mathrm{m}$ into the bulk. Given the irregularly shaped sidewalls of the inclusions, the maximum sub-surface dimension parallel to the $x$ direction of the inclusions was larger than the lattice parameter $a=4 \mathrm{um}$; hence, internal bridging of voids occurred as had been observed in a previous wet-etching report (Tarui, et al., 1971). Figure 1(b) shows the same inclusion cropped by the dimensions of the lattice parameter, where the flat surfaces at $x= \pm 2 \mu \mathrm{m}$ indicate bridging of neighbouring inclusions. Table 1 contains the pertinent elastic, dielectric, and piezoelectric coupling constants, and density of GaAs used for the simulations in COMSOL Multiphysics. 
Table 1: GaAs material constants used in piezoelectric material library of COMSOL Multiphysics

\begin{tabular}{|c|c|c|c|c|c|c|}
\hline $\begin{array}{c}\text { Material } \\
\text { Constant }\end{array}$ & $\begin{array}{c}\text { stiffness } \\
\text { constant } \\
c_{11}(\mathrm{~Pa})\end{array}$ & $\begin{array}{c}\text { stiffness } \\
\text { constant } \\
c_{12}(\mathrm{~Pa})\end{array}$ & $\begin{array}{c}\text { stiffness } \\
\text { constant } \\
c_{44}(\mathrm{~Pa})\end{array}$ & $\begin{array}{c}\text { density, } \rho \\
\left(\mathrm{kg} / \mathrm{m}^{3}\right)\end{array}$ & $\begin{array}{c}\text { relative } \\
\text { permittivity } \\
\varepsilon_{r}\end{array}$ & $\begin{array}{c}\text { piezoelectric } \\
\text { coupling } \\
e_{14}\left(\mathrm{C} / \mathrm{m}^{2}\right)\end{array}$ \\
\hline Value & $1.19263 \mathrm{e} 11$ & $5.99859 \mathrm{e} 10$ & $5.37634 \mathrm{e} 10$ & 5307 & 12.459 & 0.139785 \\
\hline
\end{tabular}

Table 2: Summary of boundary conditions applied to the phononic crystal frequency response simulation

\begin{tabular}{|c|c|c|}
\hline Boundary Surfaces & $\begin{array}{c}\text { Linear Elastic } \\
\text { Boundary Conditions }\end{array}$ & $\begin{array}{c}\text { Electrostatic } \\
\text { Boundary Conditions }\end{array}$ \\
\hline $\begin{array}{l}\text { Vacuum-GaAs } \\
\text { Interfacial Surfaces } \\
\text { - } \quad z=10 \mu \mathrm{m} \\
\text { - } \quad \text { Inclusion Surfaces }\end{array}$ & free; traction-free & $\begin{array}{l}\text { - } \text { grounded electrode: } V=0 \\
\text { - } 0 \text { pplied potential } \\
\text { electrode: } V=1 \mathrm{~V} \\
\text { - } \text { remaining: zero charge }\end{array}$ \\
\hline $\begin{array}{l}\text { Interior Planes } \\
\text { - } z=0 \\
\text { - } x=0,80 \mu \mathrm{m}\end{array}$ & non-reflecting; absorbing & zero charge \\
\hline $\begin{array}{l}\text { Lateral Interior Planes } \\
-\quad y=0,4 \mu \mathrm{m}\end{array}$ & periodic & zero charge \\
\hline
\end{tabular}

For the phononic crystal filter response, the boundary conditions are summarized in Table 2, and an image of the computational domain is seen in Figure 2. Boundaries at the $y$-extents are periodic in order to achieve a laterally infinite crystal. There are 10 inclusions along the $x$-direction with an 18 micron region beyond the phononic crystal included for determination of the transmitted acoustic wave displacement at the surface. A tetrahedral mesh with a maximum element size of $a / 3$ was used for the domains. The phononic crystal transmission and waveguide computational domains contained 438900 and 4521904 degrees of freedom, respectively, which yielded a minimum element size of approximately $a / 33$. The simulation was also conducted where the inclusions were completely removed to normalize the filter response of the phononic crystal to simple acoustic transport across a flat surface. The frequency step size was $1 \mathrm{MHz}$ for both the phononic crystal transmission and the waveguide computations.

The waveguide simulation was based on the configuration seen in Figure 3. The boundary conditions are the same as in Table 2 except the lateral planes are located at $y=0,44 \mu \mathrm{m}$. The phononic waveguide 
consisted of an array 5 inclusions laterally and 10 inclusions along either side of a linear defect, which was a line of removed inclusions. In order to produce a funnel shaped waveguide opening, point defects were also added; two inclusions and one inclusion were removed from the first and second rows of holes, respectively on either side of the linear defect.
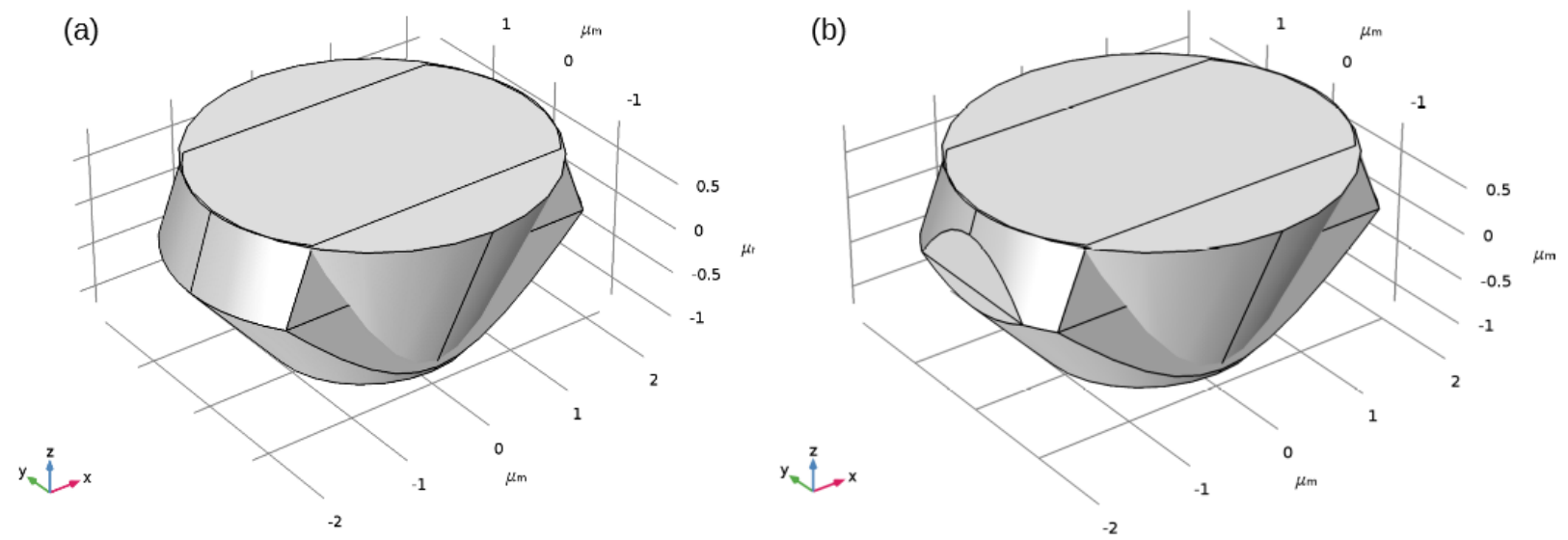

Figure 1: The phononic crystal inclusion geometry. The stretched cones are seen as elongated along the x-direction. These two cones are also truncated along the y-direction. The third cone extends in the y-direction. The full inclusion is shown in (a) while (b) represents the inclusion cropped by the width of the lattice parameter $a=4 \mu m$ in the $x$-direction. 


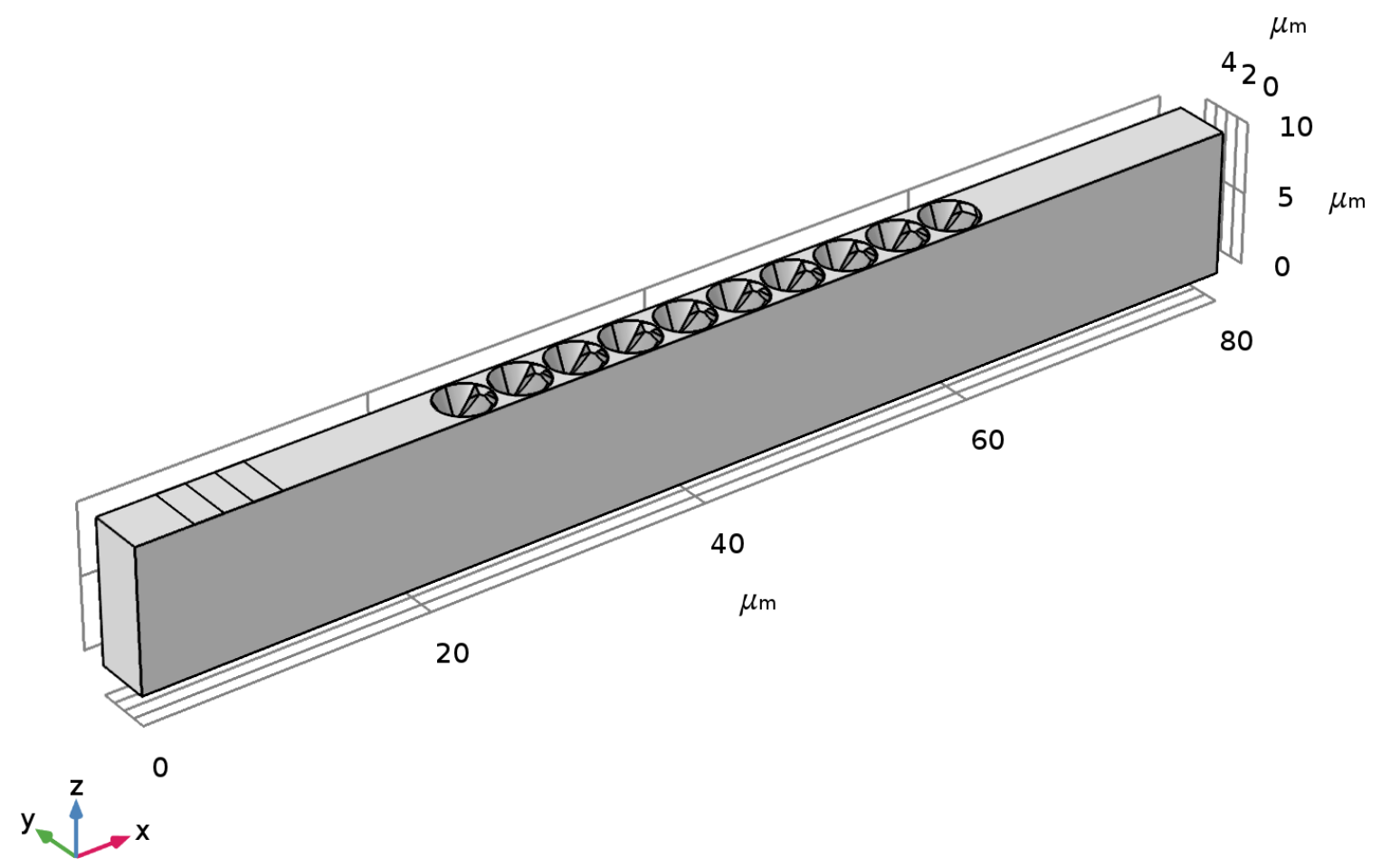

Figure 2: The computational domain in determining the phononic crystal frequency response.

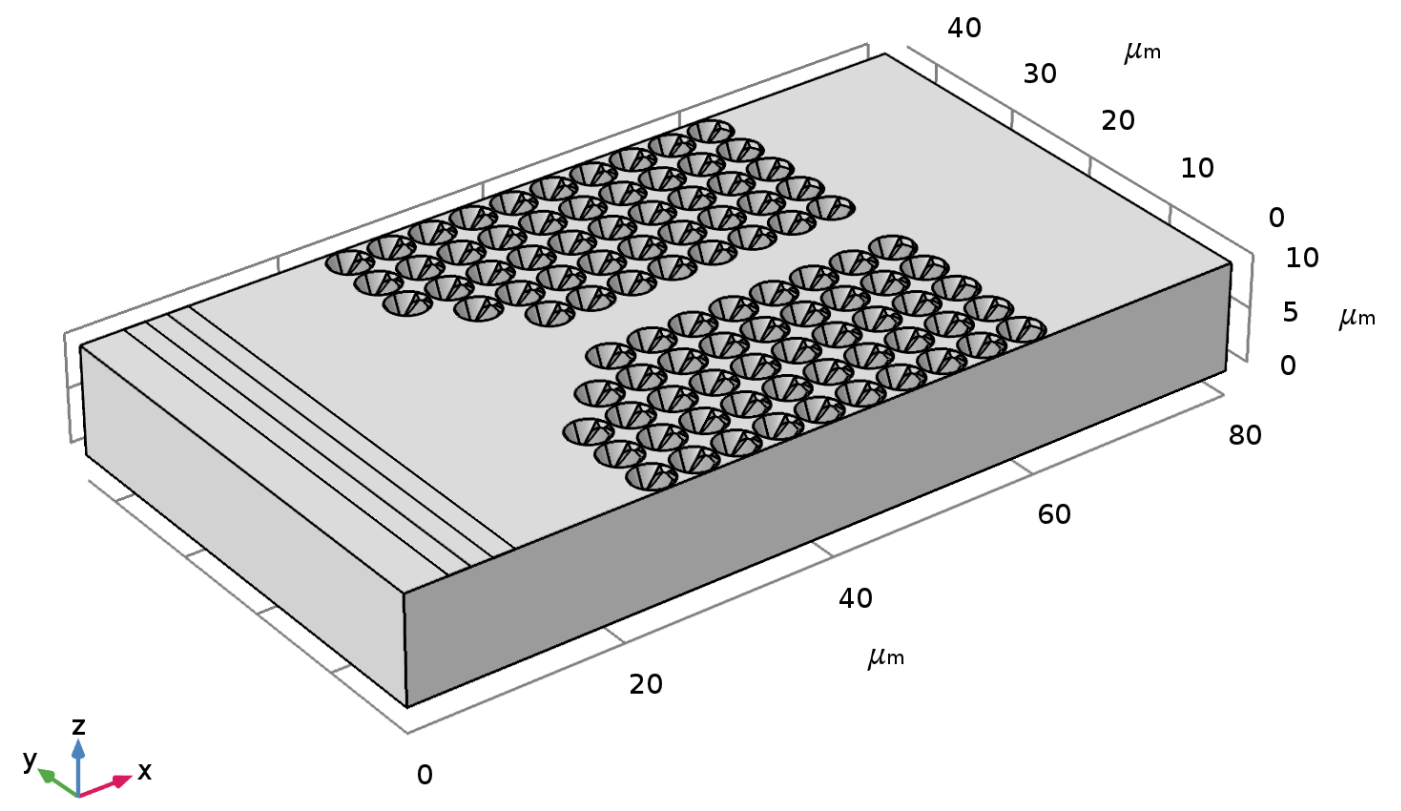

Figure 3: The waveguide computational domain. The surface is on the $z=10$ micron plane. The two surface electrodes are seen boldly outlined between $x=0$ and 20 microns. 
Both the SAW transducer as well as the phononic crystal were fabricated using optical photolithography on a (001)-oriented GaAs semi-insulating substrate. The phononic crystal waveguides were first produced using an array of circular holes with a four micron pitch and a 0.45 filling fraction on the photo mask. The L1 defect waveguide is 24 periods long $(96 \mu \mathrm{m})$ with an additional stepped entrance (and exit) where 2 and 1 holes were removed on either side of the waveguide in the transition from the unprocessed surface. After photolithographic development of the circular features, the sample underwent a wet-etch process in a solution of 1:10 of hydrogen peroxide to citric acid and 30 grams of monohydrate citric acid added to 30 $\mathrm{mL}$ of de-ionized water. The solution was applied for 8 minutes and subsequently rinsed to halt further etching. Due to the wet etch procedure, the final surface filling fraction was approximately $0.6 \pm 0.1$. Figure 4 shows an image of the fabricated phononic crystal waveguide resulting from the reflected laser intensity during the interferometry measurement. After cleaning the resist from the phononic crystal, the interdigitated transducer (IDT) was fabricated. The IDT was designed as single finger transducer with a 0.5 metallization ratio and $1.73 \mu \mathrm{m}$ linewidths to create SAWs with wavelengths of $6.92 \mu \mathrm{m}$. Using the lift-off technique, $40 \mathrm{~nm}$ thick aluminum lines formed the transducer electrodes and contact pads. The transducer provides SAWs with an approximate $2 \mathrm{MHz}$ bandwidth about a centre frequency near $410 \mathrm{MHz}$. This corresponds to a SAW velocity of $2840 \mathrm{~m} / \mathrm{s}$, which is in the range typical for SAWs in GaAs at room temperature. The IDTs were placed in the region $x<0$ w.r.t. the axis labelled in Figure 4 and Figure 9. We use surface scanning Sagnac interferometry to measure the vertical displacement amplitude. Surface scanning Sagnac interferometry is an established technique for investigating SAWs (Tachizaki, et al., 2006) and has been used to study SAWs in two-dimensional phononic crystals with finite thickness inclusions (Profunser, et al., 2006), (Petrus, et al., 2014). The surface scanning Sagnac interferometer used in this study was augmented by frequency modulating the $410.344 \mathrm{MHz}$ RF signal with a small $5 \mathrm{MHz}$ deviation to achieve on-off keying response (Mathew, 2009) (Azodi, 2013). The resulting signal is a time-averaged vertical displacement of the GaAs surface. 


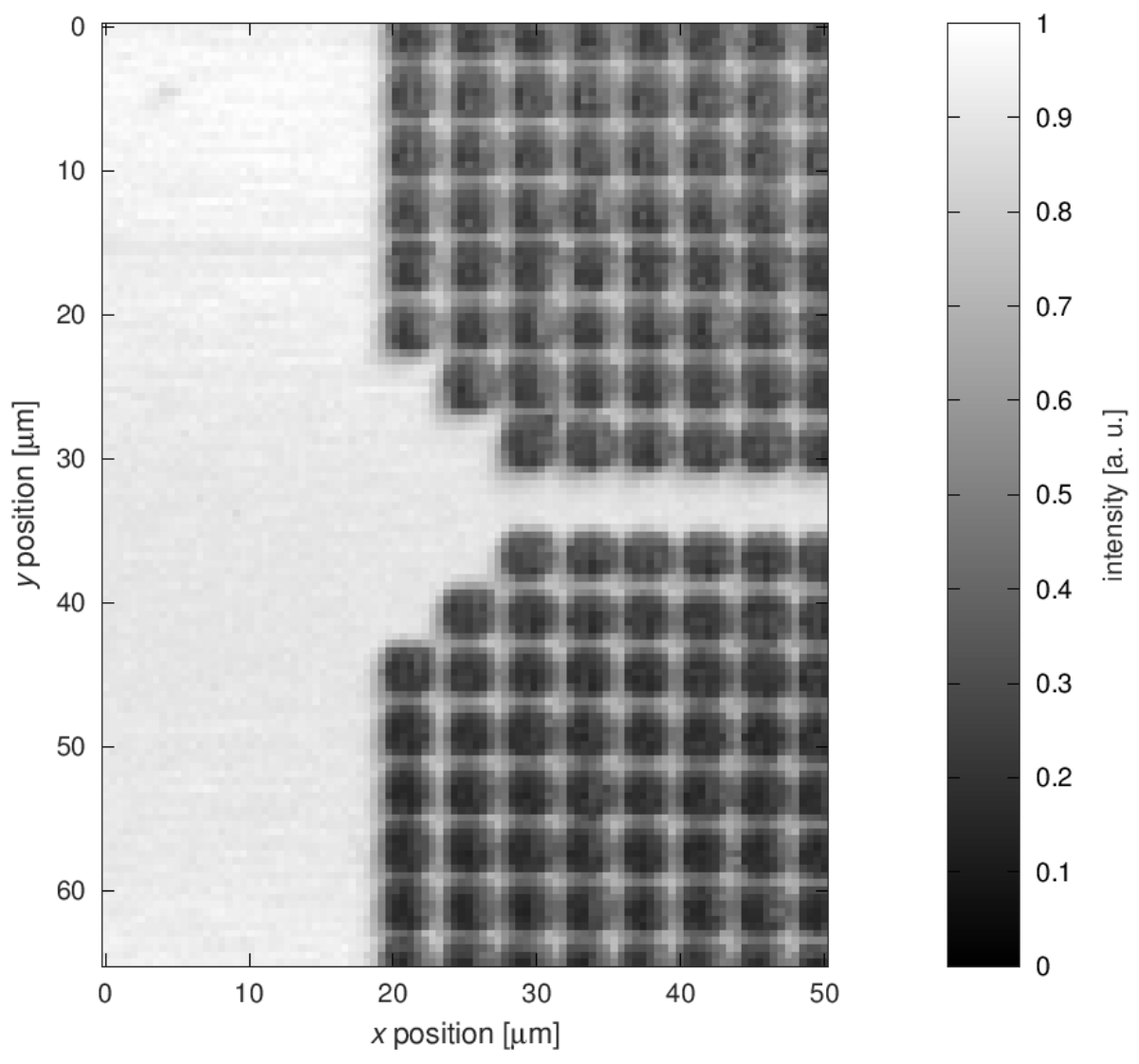

Figure 4: Image of the phononic crystal waveguide device studied by interferometry. This optical intensity is obtained concurrently with the interferometric signal.

\section{Results \& Discussion}

To first identify the stopband of the phononic crystal lattice, the transmission of the SAW across the surface was modelled for the device presented in the previous section. The simulated transmission was then quantified as the vertical displacement amplitude at the surface of the acoustic wave. Figure 5(a) shows the transmission for two configurations: the upper curve is a control of the bare substrate with no inclusions, and the lower curve reflect the effect of the shallow holes. These two curves were calculated by integrating the respective vertical displacement amplitudes over the surface beyond the phononic crystal (from $x=62 \mu \mathrm{m}$ to $x=80 \mu \mathrm{m}$ ). The values of the two curves are normalized by the spectral maximum amplitude of the substrate. In the lower curve of Figure 5(a), the phononic crystal is seen to attenuate $\left|u_{z}\right|$ at all frequencies, as might be expected with the introduction of scattering centres (Wu, et al., 2016) along the path of the SAW. However, the transmitted wave through the phononic crystal is considerably less between $400 \mathrm{MHz}$ and $450 \mathrm{MHz}$. To better visualize the effect of the phononic crystal, Figure 5(b) shows the frequency response of the phononic crystal normalized by the substrate response. The stopband of the 
phononic crystal is clearly visualized, with a minimum transmission occurring at approximately $417 \mathrm{MHz}$ with a $3 \mathrm{~dB}$ bandwidth of $25 \mathrm{MHz}$. Previous two-dimensional Finite-Difference Time-Domain modelling on GaAs phononic crystals with an $8 \mu \mathrm{m}$ pitch showed a complete bandgap from 220-235 MHz (Petrus, et al., 2014), corresponding to a bandgap of $440-470 \mathrm{MHz}$ when simply scaled to a lattice parameter of $4 \mu \mathrm{m}$. Given that the current three dimensional FEM simulations use shallow holes, the determined frequency stopband is in the range of what might be expected from the previously predicted complete bandgap.

Figure 6(a) plots of the vertical displacement amplitude within the bandgap of 400 to $450 \mathrm{MHz}$ (normalized by the substrate response) averaged over the linear defect segment adjacent to the last two inclusions in the waveguide for SAW excitation frequencies. This mode has a broadband character with a local maximum at $425 \mathrm{MHz}$, but is little varied across this frequency range. To better visualize the actual transmission of the waveguide, Figure 6(b) plots the transmission relative the phononic crystal stopband response of Figure 5(b). The relative transmission demonstrates a maximum at $419 \mathrm{MHz}$ of $18 \mathrm{~dB}$ ( $3 \mathrm{~dB}$ bandwidth of $24 \mathrm{MHz}$ ) that is significantly above any transmission through the phononic crystal stopband. The broadband character of the waveguide response has been seen in previous analyses of phononic crystal waveguides using either a finite-difference time-domain analysis of pseudo-SAWs in a steel-epoxy matrix (Sun \& Wu, 2006) or using plane wave expansive methods a silicon/air geometry (Pennec, et al., 2010). Pennec et al. have also showed that the waveguide modes (and multiple modes) may even stretch through the bandgap thus supporting virtually all frequencies in the bandgap. This broadband response limits the out-of-band rejection to essentially zero as the waveguide simply continues to transmit acoustic power for those frequencies in the stopband while having the same transmission response as the phononic crystal out of the stopband. A small frequency dependence of the transmission response of the GaAs waveguide is present, although it reveals more of a slightly varying amplitude transmission band (with transmission characteristics well above the phononic crystal stopband) rather than distinct, finite modes. 
(a)

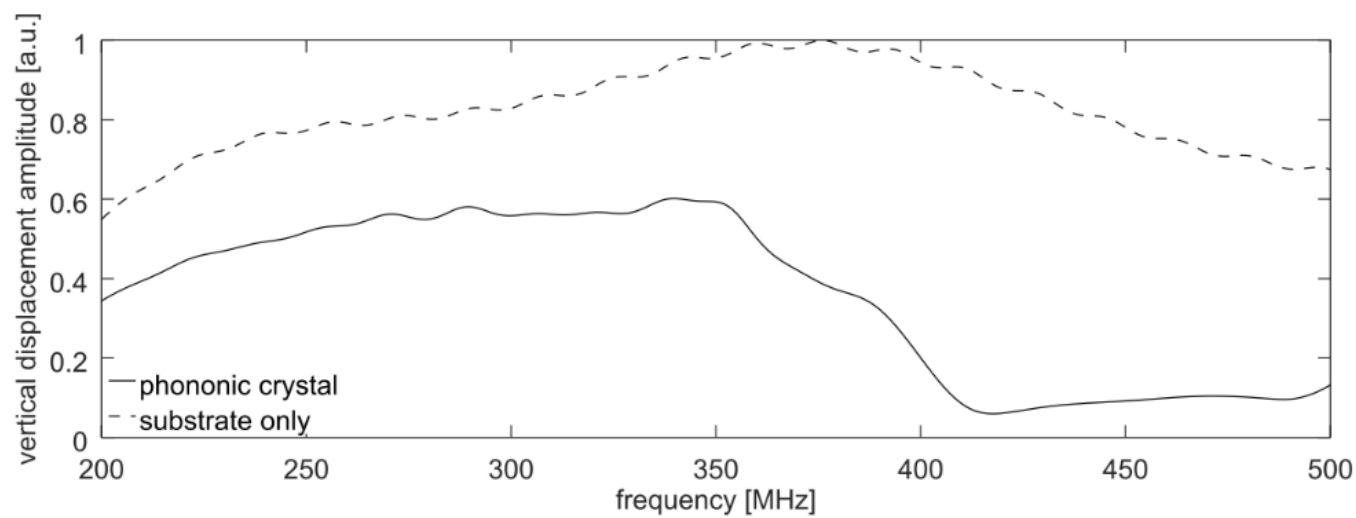

(b)

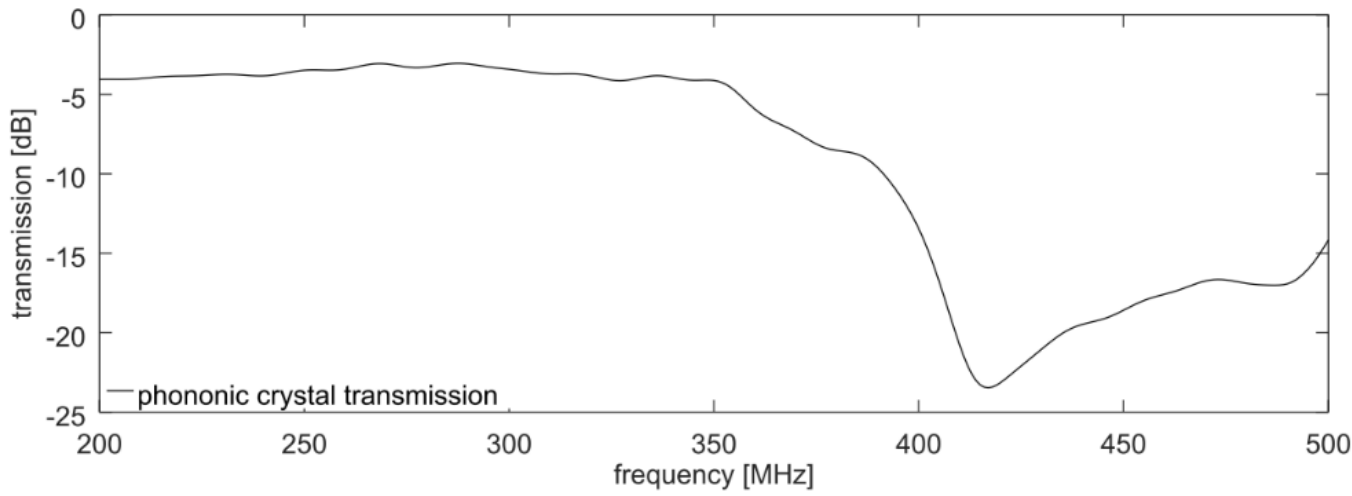

Figure 5: (a) Simulation-based vertical displacement magnitudes integrated over the surface beyond the phononic crystal for both the bare substrate and for the phononic crystal; (b) Simulation-based phononic crystal response relative to the substrate response.

(a)

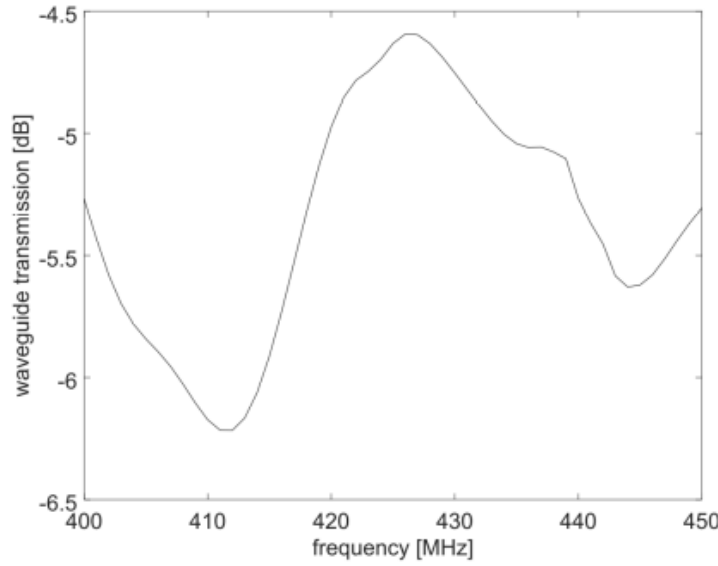

(b)

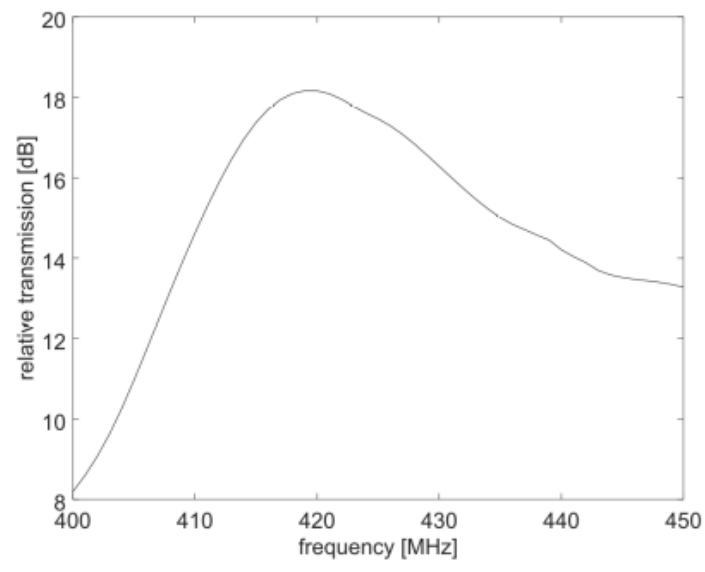

Figure 6: Simulation-based vertical displacement component amplitude spectrum averaged over the linear defect surface area adjacent to the last two inclusions. The area of integration comprises an $8 \mu m$ by $4 \mu m$ surface area of the linear defect. (a) The response is relative to the bare substrate behaviour, and (b) is the same curve with the complete phononic crystal response curve subtracted to provide the relative transmission of the waveguide. 
Figure 7 depicts an instantaneous snapshot of the real part of the vertical displacement amplitude from the GaAs surface. The geometry is the same used for the frequency response of the transmission, but the incoming SAW is excited at a single frequency of $410.344 \mathrm{MHz}$. This frequency was best mimic the frequency used in the experiment while still being near the maximum of the waveguide relative transmission band. At this frequency, Figure 7 shows that a wave is indeed evident inside the waveguide with little coupling into the adjacent phononic crystal. Additionally, when the SAW emerges from the waveguide, it diffracts similar to a point-like emitter, indicating that the wave motion was well confined by the waveguide (Benchabane, et al., 2015). This supports the notion that the waveguide is operating as expected with the bandgap of the phononic crystal confining the SAW during transmission.

To better compare with the experimental measurement, Figure 8 shows the time-integrated displacement amplitude under the same conditions as Figure 7. As the SAW impinges on the phononic crystal and entrance to the waveguide, standing wave nodes and anti-nodes are visible as a result of the reflection of the SAW from the edge of the phononic crystal and the funnel entrance. Within the waveguide, the amplitude shows some modulation along the linear defect, but there exists a relatively uniform energy density in the first few periods of the waveguide. Although it weakens, the continuous integrated displacement indicates a significant travelling wave component within the waveguide. As with Figure 7, the SAW diffracts as it exits the waveguide and travels on the free surface.

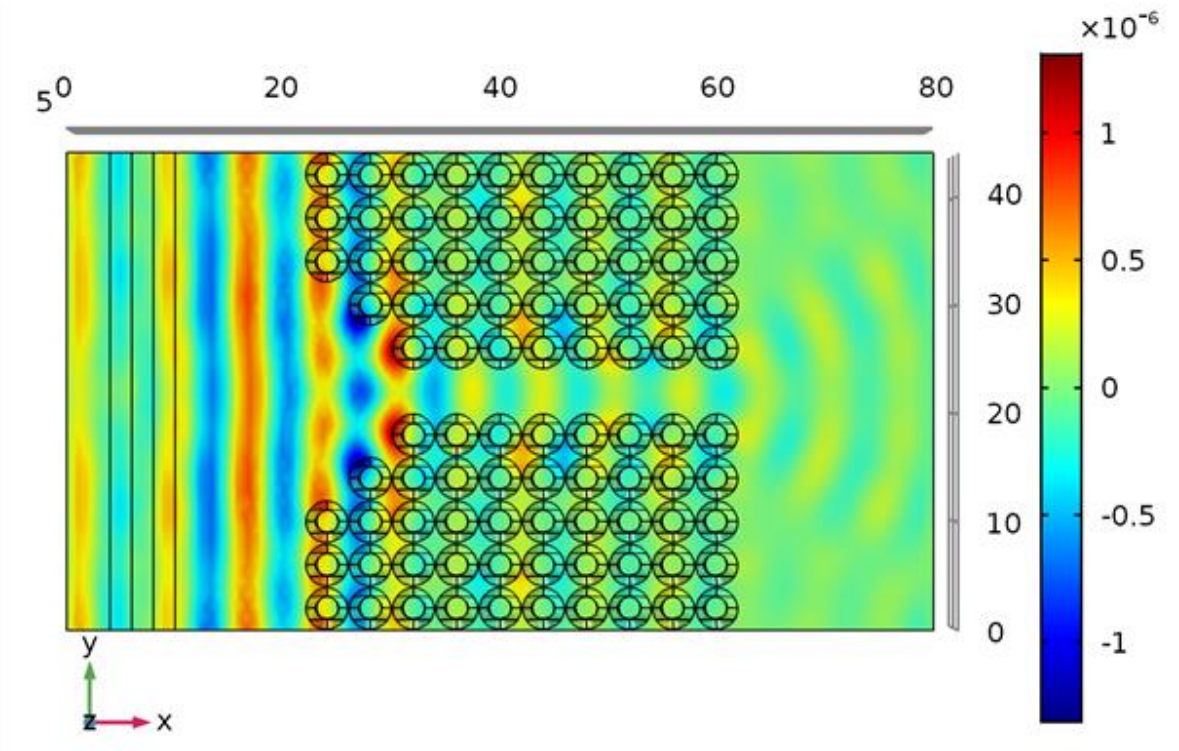

Figure 7: The simulated surface normal displacement real component from frequency domain computation at 410.334 $\mathrm{MHz}$. 


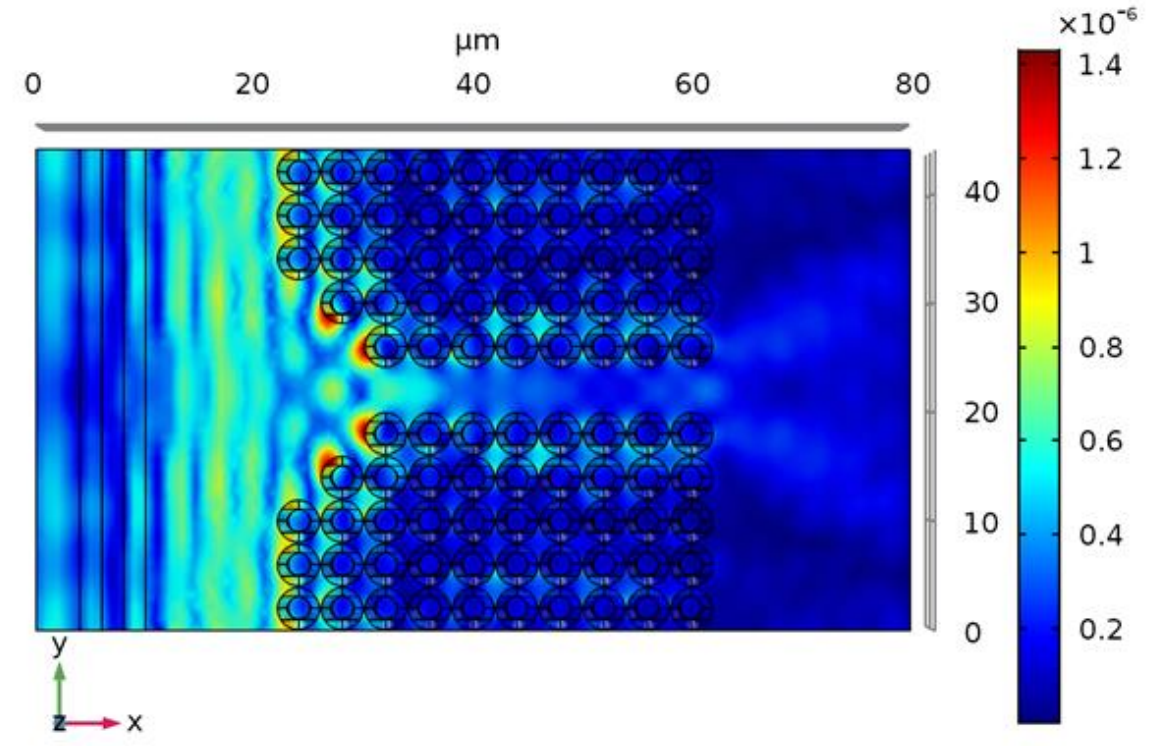

Figure 8: The simulated surface normal displacement amplitude from frequency domain computation at $410.344 \mathrm{MHz}$.

Figure 9 shows the measurement of the surface vertical displacement amplitude for the fabricated phononic crystal waveguide device operating at $410.344 \mathrm{MHz}$. Interestingly, the observed nodes and antinodes seen in front and at the entrance of the waveguide mimic those produced in the simulations. These features demonstrate that the SAWs are indeed coherently reflected from the edge of the phononic crystal in both the direction of the wave (x-direction) as well as off-axis components. The measured amplitude in the waveguide at $x>40 \mu \mathrm{m}$ is more uniform without apparent nodes and antinodes. Given that the measurement is a time-average signal, this indicates that the wave motion in this region is a travelling wave through the waveguide. Further along the waveguide (approximately $50 \mu \mathrm{m}$ ) there was no measureable acoustic signal. While these wet-etched holes do provide a platform to demonstrate waveguiding behaviour along the GaAs surface, the larger than desired attenuation is mimicked in the simulations, which have estimated decay constant of approximately $1 \mathrm{~dB} / \mathrm{a}$. The leakage of acoustic power into the phononic crystal and bulk may be caused by the Pseudo-SAW behaviour of GaAs along the [110] direction (Webster \& Carr, 1985), radiating into the substrate bulk (Wu, et al., 2016), or the coupling of multiple modes present in the bandgap (Pennec, et al., 2010). Further analysis into the origins of the decay mechanism will not be studied here.

Also observed were strong signals in the phononic crystal itself that are not as significant in the simulations. This behaviour is believed to originate from two primary factors. First, as mentioned above, the undercut of the wet etch process is believed to connect adjacent inclusions. As a result, the surface positions between inclusions are unanchored and more free to oscillate under excitation of the SAW in both the vertical and lateral directions. While the undercut is available in the simulation, the actual wet etch is more 
asymmetric resulting in larger free section in the fabricated sample. A second contribution to the large amplitudes may be due to acoustic traps related to phononic disorder caused by inclusion size variations in the wet-etch process (Wang, et al., 2009), which itself is seen by the slight asymmetry from the signal for a travelling wave in the waveguide. Disorder in phononic crystals can also shift bandgaps and waveguide bands (Li, et al., 2006) and even create new vibrational modes that are not present in the ideal phononic crystal (Wagner, et al., 2016). The FEM simulations do show non-zero vertical displacement amplitudes in the phononic crystal domains, as the wave couples to the crystal, but any localization due to disorder would not occur due to the uniform inclusions.

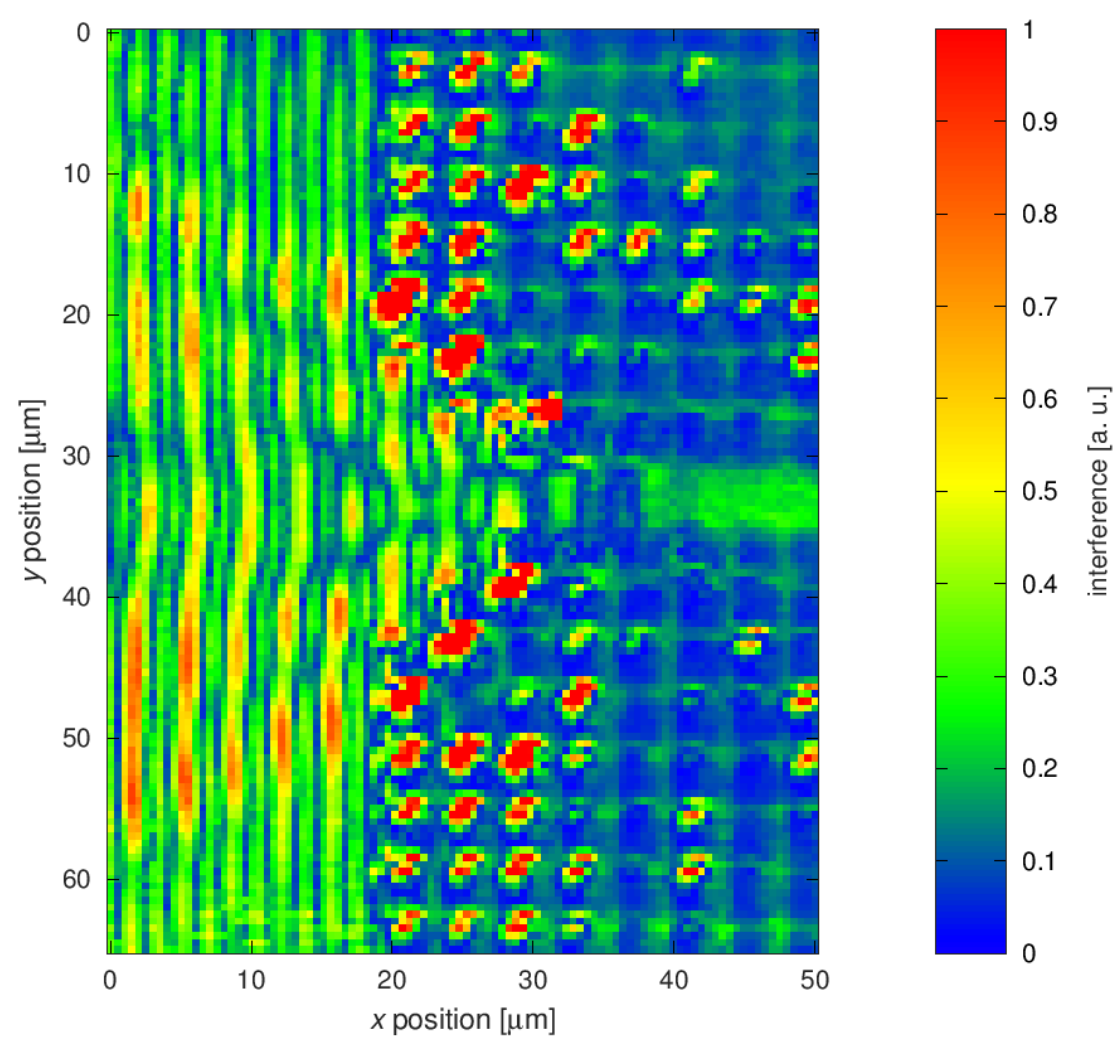

Figure 9: The measured, time-integrated interferometric signal based on surface normal displacement of the device operating at $410.344 \mathrm{MHz}$.

\section{Conclusion}

Both simulated and experimental results show the propagation of SAWs through a GaAs-based phononic crystal waveguide. The phononic crystal waveguide was composed of a square matrix of shallow, wetetched inclusions, and the non-trivial three-dimensional nature of the fabricated device was accounted for in the FEM simulations. Even though the penetration depth of the SAWs into the GaAs is much larger than the shallow holes, the inclusions are indeed shown to be sufficient to facilitate phononic crystal 
waveguiding along a bulk surface. This is the first step towards coupling phononic crystal devices with GaAs-based quantum structures to enhance the functionality and complexity of such devices. To do so, priority must be given to increase the transmission quality of the waveguide through enhanced uniformity of the crystal and its inclusions.

\section{Acknowledgements}

In acknowledgement of their contributions to this work, the authors would like to gratefully acknowledge the following: the open source GNU Octave project, whose software was used to generate plots in this work (Eaton, et al., 2017), CMC Microsystems for the provision of products and services that facilitated this research, including Design Tools, computational resources and Financial Assistance through the Micro-Nano Technologies (MNT) Facilities program; Computations were performed on resources and with support provided by the Centre for Advanced Computing (CAC) at Queen's University in Kingston, Ontario. The CAC is funded by: the Canada Foundation for Innovation, the Government of Ontario, and Queen's University; Ontario Ministry of Research and Innovation for supporting this work under Early Researcher Award ER07-04-084; Natural Science and Engineering Research Council of Canada for supporting this work under Discovery Grant RGPIN/05701-2014.

\section{References}

Azodi, G., 2013. MSc Thesis. Kingston: Queen's University.

Benchabane, S. et al., 2015. Guidance of surface waves in a micron-scale phononic crystal line-defect waveguide. Applied Physics Letters, 106(081903), pp. 1-4.

Couto Jr., O. D. D. et al., 2009. Photon anti-bunching in acoustically pumped quantum dots. Nature Photonics, 3(11), pp. 645-648.

DeSalvo, G. C., Tseng, W. F. \& Comas, J., 1992. Etch Rates and Selectivities of Citric Acid/Hydrogen Peroxide on $\mathrm{GaAs}, \mathrm{Al}_{0.3} \mathrm{Ga}_{0.7} \mathrm{As}, \mathrm{In}_{0.2} \mathrm{Ga}_{0.8} \mathrm{As}, \operatorname{In}_{0.53} \mathrm{Ga}_{0.47} \mathrm{As}, \operatorname{In}_{0.52} \mathrm{Al}_{0.48} \mathrm{As}$, and InP. J. Electrochem. Soc., 139(3), pp. 831835.

Eaton, J. W., Bateman, D., Hauberg, S. \& Wehbring, R., 2017. GNU Octave version 4.2.1 manual: a high-level interactive language for numerical computations. [Online]

Available at: https://www.gnu.org/software/octave/doc/v4.2.1/

Khelif, A. et al., 2004. Guiding and bending of acoustic waves in highly confined phononic crystal waveguides. Applied Physics Letters, 84(22). 
Li, X.-C., Liu, Z.-Y., Liang, H.-Y. \& Xiao, Q.-W., 2006. Band Gap and Waveguide States in Two-Dimensional Disorder Phononic Crystals. Chin. Phys. Lett., 23(7), pp. 1830-1833.

Mathew, R., 2009. MSc Thesis. Kingston: Queen's University.

Olsson III, R. H. \& El-Kady, I., 2009. Microfabricated phononic crystal devices and applications. Measurement Science and Technology, Volume 20, pp. 1-13.

Pennec, Y. et al., 2010. Two-dimensional phononic crystals: Examples and applications. Surface Science Reports, Volume 65, pp. 229-291.

Petrus, J. A., Mathew, R. \& Stotz, J. A. H., 2014. A GaAs Phononic Crystal With Shallow Noncylindrical Holes. IEEE Transactions on Ultrasonics, Ferroelectrics, and Frequency Control, 61(2), pp. 364-368.

Profunser, D. M., Wright, O. B. \& Matsuda, O., 2006. Imaging Ripples on Phononic Crystals Reveals Acoustic Band Structure and Bloch Harmonics. Physical Review Letters, 97(055502), pp. 1-4.

Shilton, J. M. et al., 1996. High-frequency single-electron transport in a quasi-one-dimensional GaAs channel induced by surface acoustic waves. Journal of Physics: Condensed Matter, Volume 8, pp. L531L539.

Stormer, H. L. et al., 1979. Two-dimensional electron gas at a semiconductor-semiconductor interface. Solid State Communications, 29(10), pp. 705-709.

Stotz, J. A. H., Hey, R., Santos, P. V. \& Ploog, K. H., 2005. Coherent spin transport through dynamic quantum dots. Nature Materials, Volume 4, pp. 585-588.

Sun, J.-H. \& Wu, T.-T., 2006. Guided Surface Acoustic Waves in Phononic Crystal Waveguides. s.I., IEEE Ultrasonics Symposium.

Tachizaki, T. et al., 2006. Scanning ultrafast Sagnac interferometry for imaging two-dimensional surface wave propagation. Review of Scientific Instruments, 77(043713), pp. 1-12.

Tarui, Y., Komiya, Y. \& Harada, Y., 1971. Preferential Etching and Etched Profile of GaAs. J. Electrochem. Soc., 118(1), pp. 118-122.

Wagner, M. R. et al., 2016. Two-Dimensional Phononic Crystals: Disorder Matters. Nano Letters, 16(9), pp. 5661-5668.

Wang, Y. -Z. et al., 2009. Wave localization in two-dimensional periodic systems with randomly disordered size. The European Physical Journal B, 67(4), pp. 501-505.

Webster, R. T. \& Carr, P. H., 1985. Rayleigh Waves on Gallium Arsenide. In: E. A. Ash \& E. G. S. Paige, eds. Rayleigh-Wave Theory and Application. Berlin: Springer-Verlag, pp. 122-130.

Wixforth, A., Kotthaus, J. P. \& Weimann, G., 1986. Quantum Oscillations in the Surface-Acoustic-Wave Attenuation Caused by a Two-Dimensional Electron Gas. Physical Review Letters, 56(19), pp. 2104-2106.

Wu, T.-T., Hsu, J.-C., Sun, J.-H. \& Benchabane, S., 2016. Surface Acoustic Waves in Phononic Crystals. In: A. Khelif \& A. Adibi, eds. Phononic Crystals. New York: Springer Science+Business Media, pp. 145-189.

Yudistira, D. et al., 2012. Non-radiative complete surface acoustic wave bandgap for finite-depth holey phononic crystal in lithium niobate. Applied Physics Letters, 100(061912), pp. 1-4. 
